\title{
Breast Cancer Stem Cell Mediated Epithelial-Mesenchymal Transition Targets: Hope for Breast Cancer Therapy
}

\author{
Akhilendra Kumar Maurya, Manjula Vinayak* \\ Biochemistry \& Molecular Biology Laboratory, Centre for Advanced Study in Zoology, Institute of Science, Banaras Hindu \\ University, Varanasi, India
}

*Corresponding author: Manjula Vinayak, Biochemistry \& Molecular Biology Laboratory, Centre for Advanced Study in Zoology, Institute of Science, Banaras Hindu University, Varanasi-221005, India, Tel: +91-0542-26701816, Fax: +910542368174;

E-mail: manjulavinayak@rediffmail.com

\begin{abstract}
Breast cancer is the most frequently diagnosed disease and one of the leading causes of death among woman worldwide. Treated patients often suffer from disease recurrence and metastasis due to presence of a subset of tumor cells known as breast cancer stem cells (BCSCs). Presence of BCSC is the reason for resistance and failure of therapy due to aberrant activity of aldehyde dehydrogenase, enhanced DNA damage, activation of self renewal signaling pathways and epigenetic deregulations. BCSC is a small cell population originating from normal breast stem cells, having unique characteristics such as self-renewal, high proliferation rate, ability to generate heterogeneity etc. BCSCs demonstrated aberrant activation of highly conserved signaling involved in developmental pathways such as Wnt, Notch and hedgehog as well as RTK, NF- $\kappa$ B and TGF- $\beta$ signaling. Deregulation of these signaling pathways is frequently linked to epithelial-mesenchymal transition (EMT) which plays an important role in tumor invasion and metastasis by endowing cells with a more motile and invasive phenotype. The current review will focus on aberrant signaling and regulation of EMT in BCSC; and translation of the growing knowledge into development of targeted therapies.
\end{abstract}

Keywords: Breast cancer; BCSCs; Signalling; EMT; Therapy
Received Date: July 27, 2016

Accepted Date: October 12, 2016

Published Date: October 18, 2016

Citation: Vinayak, M., et al. Breast Cancer Stem Cell Mediated Epithelial-Mesenchymal Transition Targets: Hope for Breast Cancer Therapy. (2016) Intl J Cancer Oncol 3(2): 151- 157.

DOI: $10.15436 / 2377-0902.16 .1035$

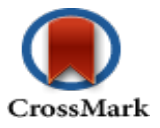

\section{Introduction}

Breast cancer is the most common cancer in woman. Conventional and targeted therapies are implicated to improve survival rates; however treatment resistance, recurrence of disease and metastasis are still major challenges in breast cancer treatment. Particular subpopulations of tumor cells are recently identified to provide resistance toward cancer therapy, namely tumor initiating cells (TICs) or cancer stem cells (CSCs) ${ }^{[1]}$. CSCs have been identified in many malignancies and are hypothesized to form clonogenic core of tumor tissues ${ }^{[2]}$. These cells could potentially originates from a more differentiated cancer cell that acquires self renewal properties, perhaps as a result of epithelial to mesenchymal transition (EMT) $)^{[3]}$. During tumor progression, cancer cells undergo EMT to acquire stem cell properties like invasiveness, dissemination and chemo-resistance. Thus, an in situ carcinoma progresses to an invasive carcinoma and cells disseminate throughout the entire body via blood and lymphatic vessels. After dissemination, cells must undergo a mesenchymal to epithelial transition (MET) to colonize in distant organs. EMT signaling is involved in development and maintenance of BCSCs (breast cancer stem cells) ${ }^{[4]}$. Therefore for targeted therapeutic achievement of BCSCs, it is needed to have better understanding of EMT signaling and also to identify targets of the pathways.

BCSCs constitutes a minority of tumor cells and characterized by expression of specific cell surface markers including $\mathrm{EpCAM}^{+}$, CD24- and $\mathrm{CD}^{-} 4^{+}$, however other markers like 
ABCG2 (ATP-binding cassette G2), CD10, CD29, CD49f, CD133 and CXCR4 (chemokine receptor type 4) have also been used to identify BCSCs ${ }^{[5]}$. BCSCs could be enriched in populations that underwent EMT due to high expression of transcription factors like Snail1, Twist1, Slug, Zebl, Zeb2, fork head box proteins $\mathrm{FOXC1}$ and $\mathrm{FOXC} 2^{[-9-9]}$. It has been shown that both normal and malignant mammary stem/progenitor cells express high level of enzyme aldehyde dehydrogenase (ALDH) ${ }^{[10]}$. Breast cancer displays frequent intra- and inter-tumor heterogeneity as the result of genetic and epigenetic alterations that often enhance the vigour of cancer cells ${ }^{[11]}$. Therefore, characterization and understanding of origin of this phenotypic and molecular diversity is paramount to improving diagnosis, prognostic and predictive biomarkers and ultimately design of therapeutic strategies.

Molecular subtypes of breast cancer have been recognized through gene expression pattern. Both ER and PR receptors were expressed in Luminal A and B subtypes; however they differ in proliferation kinetics. Basal like subtypes are frequently triple negative (ER-/PR-/HER2 ${ }^{-}$) and p53 mutant and are aggressive due to poor prognosis. Another subtype i.e. claudin low breast cancer is recognized by lack of claudin and cytokeratin, although in this case also ER and PR are not expressed. $\mathrm{ER}^{+}$patients and HER2 ${ }^{+}$patients are successfully treated with conventional targeted cancer therapy using tamoxifen and trastuzumab. However, basal like or claudin low subtype fail to respond to targeted therapy ${ }^{[12]}$. The relationship between intrinsic subtype of breast cancer and origin of BCSCs is not well defined and remains too elusive. Fragmentary reports are available to relate different subtype of breast cancer with specific markers of BCSCs. $\mathrm{CD}_{4} 4^{+} / \mathrm{CD} 24^{-}$phenotype is associated with basal and luminal subtype $\mathrm{B}$ breast cancer ${ }^{[13,14]}$. ALDH $1^{+}$phenotype is correlated with basal and HER2 ${ }^{+}$breast cancer ${ }^{[15]}$. Rare basal-like $\mathrm{ER} / \mathrm{PR}^{-} / \mathrm{K} 18^{-} / \mathrm{K}^{+}$cells expressing $\mathrm{CD} 44$ have been identified in the luminal type A breast cancer ${ }^{[16]}$. Quiescent DNA repair capabilities and over expression of drug efflux pumps make BCSCs resistant for conventional cancer therapy ${ }^{[17]}$.

Plethora of signaling pathways is involved in induction of EMT, BCSCs generation/maintenance and therapy resistance. Here is a brief review of the mechanism of EMT mediated Wnt, Notch, hedgehog, TGF- $\beta$, NF- $\kappa$ B and RTK signaling pathways as well as therapeutic resistance in breast cancer therapy.

\section{BCSCs mediated epithelial-mesenchymal transition in breast cancer metastasis}

In course of normal development, cells synchronize the process of differentiation as well as migratory pattern to gain complexity and specialization of particular cell types. Deregulation in these coordination leads to cancer. EMT plays an important and intrinsic part of normal development during organogenesis and is also recognized as a key event for metastasis ${ }^{[18-20]}$. EMT is involved in process of development like formation of primitive streak, the neural crest delamination, heart valve differentiation and lung organogenesis ${ }^{[20]}$.

Epithelial cells enhance their migratory capacity by acquiring mesenchymal state through a variety of changes like alteration of apico basal polarity, remodelling of intermediate filaments, microfilaments and intercellular adhesions by secretion of new matrix proteins and glycosamino glycans. EMT reprograms the epithelial cells to promote tumor cell metastasis. EMT de-differentiates the tumor cells and acquires motility and invasiveness to spread into distant organs and further MET reboots an epithelial program for establishment of new tumor at the sites of spreading ${ }^{[21]}$. EMT further generates CSCs exhibiting resistance to therapy. EMT plays an important role during embryogenesis and healing phenomenon in healthy cells. Loss of E-cadherin (protein necessary for cell adhesion) and increase in $\mathrm{N}$-cadherin are known to be classic marker for EMT and alteration in these marker are used to study and characterized EMT in vitro $^{[22,23]}$.

Mesenchymal phenotype required for tumor metastasis, is promoted by EMT signaling molecules produced in tumor micro environment ${ }^{[24,25]}$. TGF- $\beta$ secreted in inflamed microenvironment represses E-cadherin via stimulating the transcription factors Snail and Slug. Further, EMT activating protein Twist is activated by HIF-1, secreted in response to hypoxia. Tumorigenicity of cancer is enhanced by reduced E-cadherin, which is correlated with increased cancer grade. Protein expression in BCSCs is similar to EMT leading to decrease in E-cadherin and increase in $\mathrm{N}$-cadherin as well as increase in Slug expression ${ }^{[6,26]}$. Additionally, immortalized non tumorigenic human mammary cells undergoing EMT are enriched in CSCs markers such as $\mathrm{CD}_{4} 4^{+} / \mathrm{CD} 24^{-}$and signaling proteins SOX2, OCT $4^{[6,27]}$. The population of BCSCs is significantly increased when mammary epithelial cells transformed through HER2 overexpression undergo EMT in vitro.

BCSCs transit reversibly in two distinct development states EMT and MET due to cell plasticity ${ }^{[28-30]}$. The EMT cells are mainly quiescent and are localized at tumor invasive edge adjacent to tumor stroma. These cells express cell surface marker profile CD24- and CD44 ${ }^{+[29]}$. The second state is the MET in which cells express de-toxifying enzyme ALDH. BCSCs possessing both CSC markers CD24-, CD44 ${ }^{+}$and $\mathrm{ALDH}^{+}$show the greatest tumor initiating capacity ${ }^{[29]}$. EMT and MET are interconnected in a time dependent and tissue context dependent manner. Recently, a direct link between EMT process and gain of stem cell competence is demonstrated in cultured breast cells. In particular, it was shown that the induction of EMT program not only allows cancer cells to disseminate from the primary tumor, but also promotes their self-renewal capability ${ }^{[3]]}$. Furthermore, stemness and EMT markers in circulating tumor cells (CTCs) were associated with resistance to conventional anti-cancer therapies and treatment failure. EMT cells eventually enter into the next steps of tumor metastasis cascade, including intravasation, extravasation and formation of microscopic and macroscopic metastases in distant organs ${ }^{[9,32,33]}$. The roles of EMT to promote tumor cell dissemination are well supported by recent studies on CTCs and disseminated bone marrow tumor cells, both of which exhibited EMT and sternness characteristics ${ }^{[34,35]}$. Clinically, detection of five or more CTCs in $7.5 \mathrm{ml}$ of peripheral blood serves as an indicator of breast cancer progression and the number of CTCs in patients with metastatic breast cancer tends to be a better indicator of tumor prognosis compared to other diagnostic means $^{[36-38]}$.

\section{Breast cancer stem cells mediated Signaling pathways}

Maintenance and production of BCSCs are dependent on the microenvironment often called stem cell niche. The microenvironment involved in BCSCs consist various factors including cytokines, nutrients, $\mathrm{pH}$, oxygen pressure, extracellular matrix and immune cells ${ }^{[20,39]}$. Growth factors promote endothelial cell survival and increase the generation of invasive 
$\mathrm{BCSCs}^{[40]}$. Hypoxia affects multiple molecular pathways that causes EMT and promotes BCSCs growth leading to enhance chemo/radio-resistance ${ }^{[41,42]}$. The hypoxia inducible protein carbonic anhydrase IX (CAIX) contributes to changes in local $\mathrm{pH}$ in response to hypoxia. CAIX can promote BCSCs proliferation via activation of mTORC1 signaling ${ }^{[39]}$. Increased activity of IL- 6 and STAT3/NF- $\kappa \beta$ expression is associated with an increased number of $\mathrm{HER}^{+} \mathrm{BCSC}^{\left[{ }^{[39]}\right.}$.

Various signaling pathways having fundamental roles in regulation of self-renewal and differentiation of adult and embryonic stem cells have been linked to $\mathrm{BCSCs}^{[43]}$. Wnt, Notch and Hedgehog pathways have been implicated in resistance to therapy and an increased number of BCSCs during/after treatment. These pathways play key roles during embryonic development and homeostasis; and deregulations involved in normal breast stem cells self-renewal and differentiation result in a BCSCs phenotype. In addition, deregulation in signals associated with cell proliferation, survival and apoptosis contributes to breast cancer.

\section{Aberrant activation of developmental signaling pathways}

1. Wnt signalling: Wnt signaling pathway is crucial for embryonic development and is involved in cell fate determination, proliferation and cell migration ${ }^{[44]}$. Aberrant Wnt signaling leads to various pathological processes including cancer and neurodegenerative diseases. Wnt signaling induces and stabilizes the accumulation and translocation of $\beta$-catenin into nucleus which regulates transcription of Wnt target genes leading to build up of Snail and down-regulation of E-cadherin which ultimately induces $\mathrm{EMT}^{[45,46]}$. Canonical Wnt signaling induces the expression of Axin 2 and stabilizes Snail which promotes EMT in breast cancer ${ }^{[47,48]}$. In the non-canonical, Wnt signaling activates the ERK1/2 pathway which activates $\beta$-catenin via RTK-PI3KAKT pathway ${ }^{[27]}$. Wnt pathway target genes such as LEF1 and AXIN2 are upregulated in breast cancer ${ }^{[49]}$. Wnt signaling is important for BCSCs self renewal ${ }^{[49]}$. Wnt1 was originally identified as a proto-oncogene because it was retrieved from an oncogenic integration site of MMTV (mouse mammary tumor virus). Secretion of Wnt ligand from cells in the microenvironment has paracrine effect on the invasive edge of tumor, increasing their proliferative and invasive abilities ${ }^{[50,51]} . \Delta \mathrm{Np} 63$ is a key regulator of stem cells in both normal and malignant mammary tissues and provide direct evidence that BCSCs and normal mammary stem cells share common regulatory mechanisms ${ }^{[44]}$. Wnt signaling is required for normal mammary stem cell function and Wnt-responsive cells show enriched BCSCs in mammary gland. Natural dietary components including curcumin, resveratrol and piperine have also been found to inhibit BCSCs pool and to downregulates the Wnt pathway.

2. Notch signalling: Notch receptor has an important role in self renewal and cell fate decisions in mammary gland development ${ }^{[52-54]}$. Notch pathway is crucial in determination of cell fate and progenitor cell population as it maintains a balance between cell proliferation, differentiation and apoptosis. Early breast cancer stage frequently activates Notch signaling pathway which is correlated with poor prognosis of breast cancer ${ }^{[2,53]}$. On activation, Notch receptor is cleaved by $\gamma$-secretase, releasing the intracellular subunit NICD (Notch intracellular domain), which then translocates to nucleus, interacts with other co-factors and regulates downstream targets transcriptionally. Inhibition of Notch signaling is sufficient to reduce mammosphere formation in vitro ${ }^{[55]}$. Notch signaling requires coordination with other signals like Notch to promote EMT. TGF- $\beta$ increases Notch activity through Smad3, subsequently promoting Slug expression which suppresses E-cadherin ${ }^{[56]}$. Cross-talk of Wnt and Notch pathway is needed for tumorigenic phenotype ${ }^{[57]}$. Transcriptional factors like Sox2, Oct4 and Nanog act as master regulators of pluripotency. Sox 4 induces changes in EMT process, accompanied by an enhanced number of cells with a CD44 $/ \mathrm{CD} 24^{-}$phenotype and higher invasion and mobility of cancer cells in vivo and in vitro.

3. Hedgehog signalling: Hedgehog signaling functions in multiple tissue/cell types in developing embryo to direct organogenesis, including the ventral-dorsal pattern formation in the neural tube and anterior-posterior pattern formation in the limb. Hedgehog was first identified in a genetic screen for genes required for Drosophila's embryonic patterning. Hedgehog is an embryonic development organizer pathway that activates Gli1 and Ptch1 positive modulators of hedgehog pathway, leading to BCSCs maintenance ${ }^{[33]}$. GLI1 is a downstream mediator of Hedgehog signaling which is required for BCSCs self-renewal and tumor initiation.

\section{TNF- $\alpha / N F-\kappa B$ signaling}

$\mathrm{TNF}-\alpha$ is a pro-inflammatory cytokine involved in inflammation, immunity, cellular homeostasis and tumor progression $^{[28,58,59]}$. It activates NF- $\kappa \mathrm{B}$ which induces transcription factors associated with EMT, such as Snail, Slug, Twist, ZEB1 and ZEB2 ${ }^{[60,61]}$. NF- $\kappa B$ controls the expression of a variety of cytokines particularly IL-6 and IL-8, which are closely associated with CSC function. NF- $\mathrm{BB}$ activation is essential for BCSCs and inhibition of NF-kB activation exerts profound effects on normal breast development ${ }^{[62]}$.

\section{Transforming growth factor- $\beta$ (TGF- $\beta$ ) signaling}

TGF- $\beta$ is a potent mediator of growth inhibition in a variety of cell types via inhibition of c-Myc expression and plays a crucial role in tissue regeneration, cell differentiation, embryonic development and regulation of immune system ${ }^{[63]}$. TGF- $\beta$ suppresses tumor growth during early stage of tumor progression. EMT response declined the growth inhibitory response of TGF- $\beta^{[28]}$. TGF- $\beta$ and its receptors regulate transcription of various EMT regulators including Snail, Slug and Twist ${ }^{[28]}$. Crosstalk of TGF- $\beta$ with other signaling pathways like Notch, Wnt, $\mathrm{NF}-\kappa \mathrm{B}$ and RTK is involved in the induction of EMT ${ }^{[64,65]}$.

\section{Receptor Tyrosine kinase (RTK) signaling}

RTK has revealed the diversity in mechanisms of their activation by growth factor ligand binding to activation of intracellular tyrosine kinase domains. Mutations in RTKs and aberrant activation of their intracellular signaling pathways have been linked to cancer ${ }^{[66-72]}$. RTKs also have been involved in EMT process and tumor cell invasion. Activation of RTKs and their trafficking proteins PI3K, MAPK etc along with TGF- $\beta$ are sufficient to regulate the EMT ${ }^{[28]}$. Crosstalk of RTK with Wnt and EGFR pathways has been reported in activation of EMT ${ }^{[28]}$.

Over expression of various signaling mediators like TGF- $\beta$, TNF- $\alpha$, Wnt, Notch and Hedgehog are established to be 
activators of EMT; and induction of EMT causes stemness leading to formation and maintenance of $\mathrm{BCSCs}^{[73]}$. Suppression of the hyper activated signaling reduces tumor violence as well as EMT process ${ }^{[74,75]}$. However, neoplastic transformation of normal stem cells is known to produce $\mathrm{BCSCs}^{[76]}$. Therefore, the process of BCSC formation and EMT is not sequential. Both processes are activated with similar abnormal signaling, and promote each other. Crosstalk between various signaling pathways exerts synergistic effects in the regulation of BCSCs. Therefore, inhibition of the crosstalk between embryonic and other signaling pathways is crucial for breast cancer therapy.

\section{Breast cancer stem cells and therapy resistance}

Breast cancer starts as a local disease, but it can metastasize to the lymph nodes and distant organs. Nowadays more than $80 \%$ of patients under treatments receive adjuvant chemotherapy, although approximately $40 \%$ of patients relapse and ultimately die of metastatic breast cancer. Tumor regrowth following chemotherapy/radiotherapy can be arrested if the re-populating cells are destroyed with a selective BCSCs targeting agent ${ }^{[77]}$.

BCSCs possess an invasive gene signature which correlates with increased metastasis and poor survival ${ }^{[30,78]}$. In mouse xenograft model of human triple negative breast cancer, the metastatic cancer cells in lungs over express BCSCs marker CD44 and are able to regenerate tumor following transplantation in immune-suppressed mice ${ }^{[79]}$. High metastatic and invasive capability of BCSCs was also found in circulation of breast cancer patients who were undergoing or had completed treatment ${ }^{[80]}$. Various studies indicated that BCSCs are relatively resistant to traditional cancer therapies including chemotherapy and ioniz- ing radiation in breast cancer cell line, primary mammary tumor cells and patient derived tumor xenografts ${ }^{[81-91]}$. Chemotherapy treatment enriches the cells that express markers of BCSCs. Increased ATP binding cassette (ABC) transporters and slow cycling nature of BCSCs serves as a potential mechanism for chemoresistance. Breast cancer resistance protein (BCRP) and Pgp were identified in MCF7 which is involved in MDR. HER2 and EGFR inhibitor Lapatinib, decreased the population of BCSCs, as HER2 overexpression has been shown to drive BCSCs activity $^{[88,92]}$.

Further, the presence of residual $\mathrm{ALDH}^{+}$cells following new adjuvant chemotherapy was found to associate with a high recurrence rate ${ }^{[93]}$. Both $\mathrm{ALDH}^{+}$and $\mathrm{EpCAM}^{+} \mathrm{CD} 24^{-} \mathrm{CD}_{4} 4^{+}$are able to enhance migration/invasion capacity, intrinsic detoxifying ability, efflux activities, DNA damage response and antioxidant defence ${ }^{[94-101]}$. Current treatment strategies and compounds targeting EMT are mainly aimed at various EMT inducing signals ${ }^{[102-107]}$. EMT also results in the resistance of the MCF7 cell line to tamoxifen.

Micro RNAs (miRNAs) are associated with normal biological processes including stem cell maintenance, differentiation and development. However, deregulation is reported in various human diseases including cancer as miRNAs act as either oncogenes or tumor suppressor genes ${ }^{[108-110]}$. The miRNAs regulate EMT via various classes such as miR-183 cluster, miR-200 clusters, miR-142, miR-221-222, miR-214 cluster and let-7. The miRNAs have a vital role in breast cancer stemness, metastasis and progression ${ }^{[77,111]}$. Targeting key EMT regulators such as ZEB1, SIP1 and SIRT1 by miR-200 family inhibits EMT process $^{[112,113]}$. Further, suppression of miR-200c expression by IL-6 has been reported in EMT progression ${ }^{[14]}$.

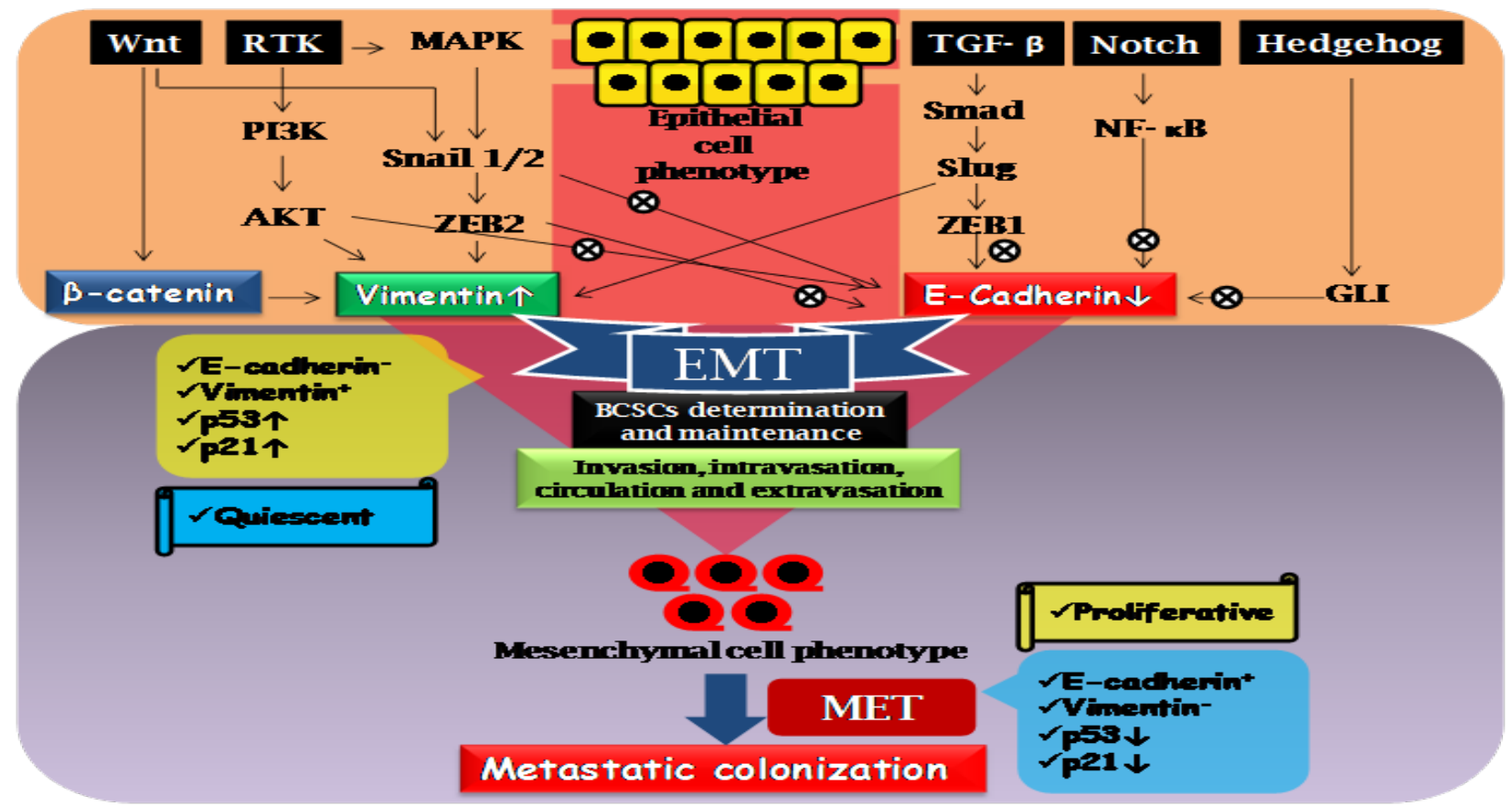

Figure 1: Molecular signaling involved in induction of EMT and regulating BCSCs in breast cancer metastasis

Aberrant activation of Wnt, RTK, TGF- $\beta$, Notch and Hedgehog signalling leads to EMT converting epithelial cells to mesenchymal cell phenotype. Transcription factors such as Snail1/2, ZEB1/2, Slug, Smad, NF-kB, GLI as well as signalling protein PI3K and AKT, promote EMT via suppression of E-cadherin and induction of Vimentin. EMT is crucial for invasion, intravasation, circulation and extravasation; and ultimately leads to colonization of metastatic cells by mesenchymal to epithelial transition (MET). The EMT cells are characterized as relatively quiescent, E cadherin negative, Vimentin positive and upregulated p53 and p21; however MET cell are proliferative, E cadherin positive, Vimentin negative having downregulated $\mathrm{p} 53$ and $\mathrm{p} 21$. 


\section{Conclusions}

The critical roles of BCSCs in breast cancer carcinogenesis highlight the demands for developing novel therapeutic strategies to eradicate this disease. Plasticity of BCSCs to alteration between EMT and MET state allows for invasion, dissemination and metastatic growth at distal organs resulting in therapeutic resistance. The plasticity could be readily identified by expression of distinct cellular markers. Targeting BCSCs mediated EMT via modulation of Wnt, Hedgehog, Notch, TNF- $\alpha /$ $\mathrm{NF}-\kappa \mathrm{B}, \mathrm{TGF}-\beta$ and RTK signaling pathways promises prevention of therapy resistance and disease recurrences. Various signaling pathways involved in breast cancer metastasis do not operate in isolation, but coordinated network is associated with phenotype of BCSCs. Modulation of Wnt, Hedgehog, Notch, TNF- $\alpha / N F-\kappa B$, TGF $-\beta$ and RTK signaling pathways might trigger surprising compensatory pathways that could neutralize the treatment effects. Therefore, in-depth understanding of the coordinated signaling network is needed to target BCSCs with specific inhibitors in combination.

Conflicts of Interest: Authors have no conflict of interest.

Acknowledgements: Research was supported by University Grants Commission (UGC), India. AKM thanks CSIR, India, for JRF \& SRF.

\section{References}

1. Iqbal, J., Chong, P.Y., Tan, P.H. Breast cancer stem cells: an update. (2013) J Clin Pathol 66(6): 485-490.

2. Vermeulen, L., Sprick, M.R., Kemper, K., et al. Cancer stem cellsold concepts, new insights. (2008) Cell Death Differ 15(6): 947-958.

3. Espinoza, I., Miele, L. Deadly crosstalk: notch signaling at the intersection of EMT and cancer stem cells. (2013) Cancer Lett 341(1): 41-45.

4. Ansieau, S. EMT in breast cancer stem cell generation. (2013) Cancer Lett 338(1): 63-68.

5. Luo, M., Brooks, M., Wicha, M.S. Epithelial-mesenchymal plasticity of breast cancer stem cells: implications for metastasis and therapeutic resistance. (2015) Curr Pharm Des 21(10):1301-1310.

6. Mani, S.A., Guo, W., Liao, M.J., et al. The epithelial mesenchymal transition generates cells with properties of stem cells. (2008) Cell 133(4): 704-715.

7. Morel, A.P., Lievre, M., Thomas, C., et al. Generation of breast cancer stem cells through epithelial-mesenchymal transition. (2008) PLoS One 3(8): e2888.

8. Moreno-Bueno, G., Portillo, F., Cano, A. Transcriptional regulation of cell polarity in EMT and cancer. (2008) Oncogene 27(55): 69586969.

9. Brabletz, T. To differentiate or not--routes towards metastasis. (2012) Nat Rev Cancer 12(6): 425-436.

10. Ginestier C, Hur MH, Charafe-Jauffret E, et al. ALDHI is a marker of normal and malignant human mammary stem cells and a predictor of poor clinical outcome. (2007) Cell stem cell 1(5): 555-567.

11. Koren, S., Bentires-Alj, M. Breast Tumor Heterogeneity: Source of Fitness, Hurdle for Therapy. (2015) Mol Cell 60(4): 537-546.

12. Pandey, S.K., Patel, D.K., Maurya, A.K., et al. Controlled release of drug and better bioavailability using poly(lactic-co-glycolic acid) nanoparticles. (2016) Int J Biol Macromol 89: 99-110.

13. Nakshatri, H., Srour, E.F., Badve, S. Breast cancer stem cells and intrinsic subtypes: controversies rage on. (2009) Curr Stem Cell Res Ther 4(1): 50-60.
14. Engelmann, K., Shen, H., Finn, O.J. MCF7 side population cells with characteristics of cancer stem/progenitor cells express the tumor antigen MUC1. (2008) Cancer Res 68(7): 2419-2426.

15. Ginestier, C., Hur, M.H., Charafe-Jauffret, E., et al. ALDH1 is a marker of normal and malignant human mammary stem cells and a predictor of poor clinical outcome. (2007) Cell Stem Cell 1(5): 555-567.

16. Horwitz, K.B., Dye, W.W., Harrell, J.C., et al. Rare steroid receptor-negative basal-like tumorigenic cells in luminal subtype human breast cancer xenografts. (2008) Proc Natl Acad Sci U S A 105(15): 5774-5779.

17. Dean, M., Fojo, T., Bates, S. Tumour stem cells and drug resistance. (2005) Nat Rev Cancer 5(4): 275-284.

18. Chakrabarti, R., Hwang, J., Andres Blanco, M., et al. Elf5 inhibits the epithelial-mesenchymal transition in mammary gland development and breast cancer metastasis by transcriptionally repressing Snail2. (2012) Nat Cell Biol 14(11):1212-1222.

19. Brabletz, T., Hlubek, F., Spaderna, S., et al. Invasion and metastasis in colorectal cancer: epithelial-mesenchymal transition, mesenchymal-epithelial transition, stem cells and beta-catenin. (2005) Cells Tissues Organs 179(1-2): 56-65.

20. Thiery, J.P., Acloque, H., Huang, R.Y., et al. Epithelial-mesenchymal transitions in development and disease. (2009) Cell 139(5): 871890.

21. Creighton, C.J., Li, X., Landis, M., et al. Residual breast cancers after conventional therapy display mesenchymal as well as tumor-initiating features. (2009) Proc Natl Acad Sci USA 106(33): 13820-13825.

22. Krawczyk, N., Meier-Stiegen, F., Banys, M., et al. Expression of stem cell and epithelial-mesenchymal transition markers in circulating tumor cells of breast cancer patients. (2014) Biomed Res Int 2014: 11.

23. Hollier, B.G., Evans, K., Mani, S.A. The epithelial-to-mesenchymal transition and cancer stem cells: a coalition against cancer therapies. (2009) J Mammary Gland Biol Neoplasia 14(1): 29-43.

24. Gao, T., Li, J.Z., Lu, Y., et al. The mechanism between epithelial mesenchymal transition in breast cancer and hypoxia microenvironment. (2016) Biomed Pharmacother 80: 393-405.

25. Foroni, C., Broggini, M., Generali, D., et al. Epithelial-mesenchymal transition and breast cancer: role, molecular mechanisms and clinical impact. (2012) Cancer Treat Rev 38(6): 689-697.

26. May, C.D., Sphyris, N., Evans, K.W., et al. Epithelial-mesenchymal transition and cancer stem cells: a dangerously dynamic duo in breast cancer progression. (2011) Breast Cancer Res 13(1): 202.

27. Wang, Y., Dong, J., Li, D., et al. Lgr4 regulates mammary gland development and stem cell activity through the pluripotency transcription factor Sox2. (2013) Stem Cells 31(9): 1921-1931.

28. Voutsadakis, I.A. The network of pluripotency, epithelial-mesenchymal transition, and prognosis of breast cancer. (2015) Breast Cancer (Dove Med Press) 7: 303-319.

29. Liu, S., Cong, Y., Wang, D., et al. Breast cancer stem cells transition between epithelial and mesenchymal states reflective of their normal counterparts. (2014) Stem Cell Rep 2(1): 78-91.

30. Pinto, C.A., Widodo, E., Waltham, M., et al. Breast cancer stem cells and epithelial mesenchymal plasticity - Implications for chemoresistance. (2013) Cancer Lett 341(1): 56-62.

31. Nieto, M.A. Epithelial plasticity: a common theme in embryonic and cancer cells. (2013) Science 342(6159):1234850.

32. Wu, Y., Sarkissyan, M., Vadgama, J.V. Epithelial-Mesenchymal Transition and Breast Cancer. (2016) J Clin Med 5(2): 13.

33. Kalluri, R., Weinberg, R.A. The basics of epithelial-mesenchymal transition. (2009) J Clin Invest 119(6): 1420-1428.

34. Watson, M.A., Ylagan, L.R., Trinkaus, K.M., et al. Isolation and molecular profiling of bone marrow micrometastases identifies TWIST1 as a marker of early tumor relapse in breast cancer patients. (2007) Clin Cancer Res 13(17): 5001-5009.

35. Raimondi, C., Gradilone, A., Naso, G., et al. Epithelial-mesenchymal transition and stemness features in circulating tumor cells from breast cancer patients. (2011) Breast Cancer Res Treat 130(2): 449-455. 
36. Pantel, K., Alix-Panabieres, C., Riethdorf, S. Cancer micrometastases. (2009) Nat Rev Clin Oncol 6(6): 339-351.

37. Yu, M., Stott, S., Toner, M., et al. Circulating tumor cells: approaches to isolation and characterization. (2011) J Cell Biol 192(3): 373-382. 38. Baccelli, I., Schneeweiss, A., Riethdorf, S., et al. Identification of a population of blood circulating tumor cells from breast cancer patients that initiates metastasis in a xenograft assay. (2013) Nat Biotechnol 31(6): 539-544.

39. Bozorgi, A., Khazaei, M., Khazaei, M.R. New Findings on Breast Cancer Stem Cells: A Review. (2015) J Breast Cancer 18(4): 303-312. 40. Kuonen, F., Secondini, C., Rüegg, C. Molecular pathways: emerging pathways mediating growth, invasion, and metastasis of tumors progressing in an irradiated microenvironment. (2012) Clin Cancer Res 18(19): 5196-5202.

41. Batchelor, T.T., Gerstner, E.R., Emblem, K.E., et al. Improved tumor oxygenation and survival in glioblastoma patients who show increased blood perfusion after cediranib and chemoradiation. (2013) Proc Natl Acad Sci U S A 110(47):19059-19064.

42. Pece, S., Tosoni, D., Confalonieri, S., et al. Biological and molecular heterogeneity of breast cancers correlates with their cancer stem cell content. (2010) Cell 140(1): 62-73.

43. Fazilaty, H., Gardaneh, M., Bahrami, T., et al. Crosstalk between breast cancer stem cells and metastatic niche: emerging molecular metastasis pathway? (2013) Tumour Biol 34(4): 2019-2030.

44. Chakrabarti, R., Wei, Y., Hwang, J., et al. $\Delta$ Np63 promotes stem cell activity in mammary gland development and basal-like breast cancer by enhancing Fzd7 expression and Wnt signalling. (2014) Nat Cell Biol 16(10):1004-15, 1-13.

45. Wang, Y., Zhou, B.P. Epithelial-mesenchymal transition in breast cancer progression and metastasis. (2011) Chin J Cancer 30(9): 603611.

46. Reya, T., Clevers, H. Wnt signalling in stem cells and cancer. (2005) Nature 434(7035): 843-850.

47. Kotiyal, S, Bhattacharya S. Breast cancer stem cells, EMT and therapeutic targets. (2014) Biochem Biophys Res Commun 453(1):112-116. 48. Yook, J.I., Li, X.Y., Ota, I., et al. A Wnt-Axin2-GSK3bold beta cascade regulates Snaill activity in breast cancer cells. (2006) Nat Cell Biol 8(12): 1398-1406.

49. Lamb, R., Ablett, M.P., Spence, K., et al. Wnt pathway activity in breast cancer sub-types and stem-like cells. (2013) PLoS One 8(7): e67811.

50. Wolfson, B., Eades, G., Zhou, Q. Adipocyte activation of cancer stem cell signaling in breast cancer. (2015) World J Biol Chem 6(2): 39-47.

51. Fodde, R., Brabletz, T. Wnt/beta-catenin signaling in cancer stemness and malignant behavior. (2007) Curr Opin Cell Biol 19(2): 150158.

52. Farnie, G., Clarke, R.B. Mammary stem cells and breast cancerrole of Notch signalling. (2007) Stem Cell Rev 3(2): 169-175.

53. Guo, S., Liu, M., Gonzalez-Perez, R.R. Role of Notch and its oncogenic signaling crosstalk in breast cancer. (2011) Biochim Biophys Acta 1815(2): 197-213.

54. Dontu, G., Jackson, K.W., McNicholas, E., et al. Role of Notch signaling in cell-fate determination of human mammary stem/progenitor cells. (2004) Breast Cancer Res 6(6): 605-615.

55. Zhao, D., Mo, Y., Li, M.T., et al. NOTCH-induced aldehyde dehydrogenase 1A1 deacetylation promotes breast cancer stem cells. (2014) J Clin Invest 124(12): 5453-5465.

56. Leong, K.G., Niessen, K., Kulic, I., et al. Jagged1-mediated Notch activation induces epithelial-to-mesenchymal transition through Slug-induced repression of E-cadherin. (2007) J Exp Med 204(12): 2935-2948.

57. Collu, G.M., Brennan, K. Cooperation between Wnt and Notch signalling in human breast cancer. (2007) Breast Cancer Res 9(3): 105. 58. Mishra, S., Vinayak, M. Ellagic acid checks lymphoma promotion via regulation of PKC signaling pathway. (2013) Mol Biol Rep 40(2):1417-1428.
59. Das, L., Vinayak, M. Anti-carcinogenic action of curcumin by activation of antioxidant defence system and inhibition of NF-kappaB signalling in lymphoma-bearing mice. (2012) Biosci Rep 32(2): 161-170. 60. Min, C., Eddy, S.F., Sherr, D.H., et al. NF-kappa B and epithelial to mesenchymal transition of cancer. (2008) J Cell Biochem 104(3): 733-744.

61. Wu, Y., Deng, J., Qiu, S., et al. Stabilization of snail by NF-kappa $\mathrm{B}$ is required for inflammation-induced cell migration and invasion. (2009) Cancer Cell 15(5): 416-428.

62. Yamamoto, M., Taguchi, Y., Ito-Kureha, T., et al. NF-kB non-cell-autonomously regulates cancer stem cell populations in the basal-like breast cancer subtype. (2013) Nat Commun 4: 2299.

63. Mishra, S., Vinayak, M. Anti-carcinogenic action of ellagic acid mediated via modulation of oxidative stress regulated genes in Dalton lymphoma bearing mice. (2011) Leuk Lymphoma 52(11): 2155-2161. 64. Gal, A., Sjoblom, T., Fedorova, L., et al. Sustained TGF b exposure suppresses Smad and non-Smad signalling in mammary epithelial cells, leading to EMT and inhibition of growth arrest and apoptosis. (2008) Oncogene 27(9): 1218-1230.

65. Janda, E., Lehmann, K., Killisch, I., et al. Ras and TGFb cooperatively regulate epithelial cell plasticity and metastasis: dissection of Ras signaling pathways. (2002) J Cell Biol 156(2): 299-313.

66. Lemmon, M.A., Schlessinger, J. Cell Signaling by Receptor Tyrosine Kinases. (2010) Cell 141(7): 1117-1134.

67. Maurya, A.K., Vinayak, M. Modulation of PKC signaling and induction of apoptosis through suppression of reactive oxygen species and tumor necrosis factor receptor 1 (TNFR1): key role of quercetin in cancer prevention. (2015) Tumour Biol 36(11): 8913-8924.

68. Maurya, A.K., Vinayak, M. Anticarcinogenic action of quercetin by downregulation of phosphatidylinositol 3-kinase (PI3K) and protein kinase $\mathrm{C}(\mathrm{PKC})$ via induction of $\mathrm{p} 53$ in hepatocellular carcinoma (HepG2) cell line. (2015) Mol Biol Rep 42(9):1419-1429.

69. Maurya, A.K., Vinayak, M. Abstract A07: Decline in the growth of murine T-cell lymphoma via modulation of PI3K signaling pathway: key role of quercetin and PI-103. (2015) Mol Cancer Ther 14(7): A07. 70. Maurya, A.K., Vinayak, M. Quercetin regresses Dalton's lymphoma growth via suppression of PI3K/AKT signaling leading to upregulation of p53 and decrease in energy metabolism. (2015) Nutr Cancer 67(2): 354-363.

71. Maurya, A.K., Vinayak, M. PI-103 attenuates PI3K-AKT signaling and induces apoptosis in murine T-cell lymphoma. (2016) Leuk Lymphoma 1-9.

72. Maurya, A.K., Vinayak, M. PI-103 and quercetin attenuate PI3KAKT signaling pathway in T- cell lymphoma exposed to hydrogen peroxide. (2016) PLoS One 11(8): e0160686.

73. Li, J., Zhou, B.P. Activation of $\beta$-catenin and Akt pathways by Twist are critical for the maintenance of EMT associated cancer stem cell-like characters. (2011) BMC Cancer 11: 49.

74. Hollier, B.G., Tinnirello, A.A., Werden, S.J., et al. FOXC2 expression links epithelial-mesenchymal transition and stem cell properties in breast cancer. (2013) Cancer Res 73(6):1981-1992.

75. Asiedu, M.K., Ingle, J.N., Behrens, M.D., et al. TGFbeta/TNF(alpha)-mediated epithelial-mesenchymal transition generates breast cancer stem cells with a claudin-low phenotype. (2011) Cancer Res 71(13): 4707-4719.

76. Serakinci, N., Guldberg, P., Burns, J.S., et al. Adult human mesenchymal stem cell as a target for neoplastic transformation. (2004) Oncogene 23(29): 5095-5098.

77. Liu, S., Clouthier, S.G., Wicha, M.S. Role of microRNAs in the regulation of breast cancer stem cells. (2012) J Mammary Gland Biol Neoplasia 17(1):15-21.

78. Liu, R., Wang, X., Chen, G.Y., et al. The prognostic role of a gene signature from tumorigenic breast-cancer cells. (2007) N Eng J Med 356(3): 217-226.

79. Liu, H., Patel, M.R., Prescher, I.A., et al. Cancer stem cells from human breast tumors are involved in spontaneous metastases in orthotopic mouse models. (2010) Proc Natl Acad Sci USA 107(42): 18115-18120. 
80. Patel, S.A., Ramkissoon, S.H., Bryan, M., et al. Delineation of breast cancer cell hierarchy identifies the subset responsible for dormancy. (2012) Sci Rep 2: 906.

81. Lagadec, C., Vlashi, E., Della Donna, L., et al. Survival and self-renewing capacity of breast cancer initiating cells during fractionated radiation treatment. (2010) Breast Cancer Res 12(1): R13.

82. Karimi-Busheri, F., Rasouli-Nia, A., Mackey, J.R., et al. Senescence evasion by MCF-7 human breast tumor-initiating cells. (2010) Breast Cancer Res 12(3): R31.

83. Fillmore, C.M., Kuperwasser, C. Human breast cancer cell lines contain stem-like cells that self-renew, give rise to phenotypically diverse progeny and survive chemotherapy. (2008) Breast Cancer Res 10(2): R25.

84. Shafee, N., Smith, C.R., Wei, S., et al. Cancer stem cells contribute to cisplatin resistance in Brca1/p53-mediated mouse mammary tumors. (2008) Cancer Res 68(9): 3243-3250.

85. Woodward, W.A., Chen, M.S., Behbod, F., et al. WNT/beta-catenin mediates radiation resistance of mouse mammary progenitor cells. (2007) Proc Natl Acad Sci USA 104(2): 618-623.

86. Diehn, M., Cho, R.W., Lobo, N.A., et al. Association of reactive oxygen species levels and radioresistance in cancer stem cells. (2009) Nature 458(7239): 780-783.

87. Zielske, S.P., Spalding, A.C., Wicha, M.S., et al. Ablation of breast cancer stem cells with radiation. (2011) Transl Oncol 4(4): 227-233.

88. Li, X., Lewis, M.T., Huang, J., et al. Intrinsic resistance of tumorigenic breast cancer cells to chemotherapy. (2008) J Natl Cancer Institute 100(9): 672-679.

89. Dave, B., Mittal, V., Tan, N.M., et al. Epithelial-mesenchymal transition, cancer stem cells and treatment resistance. (2012) Breast Cancer Res 14(1): 202.

90. Nandy, S.B., Gangwani, L., Nahleh, Z., et al. Recurrence and metastasis of breast cancer is influenced by ovarian hormone's effect on breast cancer stem cells. (2015) Future Oncol 11(6): 983-995.

91. Creighton, C.J., Chang, J.C., Rosen, J.M. Epithelial-mesenchymal transition (EMT) in tumor-initiating cells and its clinical implications in breast cancer. (2010) J Mammary Gland Biol Neoplasia 15(2): 253-260. 92. Korkaya, H., Paulson, A., Iovino, F., et al. ITER2 regulates the mammary stem/progenitor cell population driving tumorigenesis and invasion. (2008) Oncogene 27(47): 6120-6130.

93. Alamgeer, M., Ganju, F., Kumar, B., et al. Changes in aldehyde dehydrogenase-1 expression during neoadjuvant chemotherapy predict outcome in locally advanced breast cancer. (2014) Breast Cancer Res 16(2): R44.

94. Liu, S., Ginestier, C., Ou, S.J., et al. Breast cancer stem cells are regulated by mesenchymal stem cells through cytokine networks. (2011) Cancer Res 71(2): 614-624.

95. Moreb, J.S., Maccow, C., Schweder, M., et al. Expression of antisense RNA to aldehyde dehydrogenase class-1 sensitizes tumor cells to 4-hydroperoxycyclophosphamide in vitro. (2000) J Pharmacol Exp Ther 293(2): 390-396.

96. Britton, K.M., Eyre, R., Harvey, I.J., et al. Breast cancer, side population cells and ABCG2 expression. (2012) Cancer Lett 323(1): 97-105. 97. Nakanishi, T., Chumsri, S., Khakpour, N., et al. Side-population cells in luminal-type breast cancer have tumour-initiating cell properties, and are regulated by HER2 expression and signalling. (2010) Br J Cancer 102(5): 815-826.

98. Yin, H., Glass, J. The phenotypic radiation resistance of CD44+/ CD24(-or low) breast cancer cells is mediated through the enhanced activation of ATM signaling. (2011) PloS One 6(9): e24080.

99. Zhang, M., Atkinson, R.L., Rosen, J.M. Selective targeting of radiation-resistant tumor-initiating cells. (2010) Proc Natl Acad Sci USA 107(8): 3522-3527.
100. Hiraga, T., Ito, S., Nakamura, H. Cancer stem-like cell marker CD44 promotes bone metastases by enhancing tumorigenicity, cell motility, and hyaluronan production. (2013) Cancer Res 73(13): 41124122.

101. Brown, R.L., Reinke, L.M., Damerow, M.S., et al. CD44 splice isoform switching in human and mouse epithelium is essential for epithelial-mesenchymal transition and breast cancer progression. (2011) J Clin Invest 121(3): 1064-1074.

102. Creighton, C.J., Li, X., Landis, M., et al. Residual breast cancers after conventional therapy display mesenchymal as well as tumor-initiating features. (2009) Proc Nati Acad Sci USA 106(33): 13820-13825. 103. Tsai, J.H., Yang, J. Epithelial-mesenchymal plasticity in carcinoma metastasis. (2013) Genes Dev 27(20): 2192-2206.

104. Reka, A.K., Kuick, R., Kurapati, H., et al. Identifying inhibitors of epithelial-mesenchymal transition by connectivity map-based systems approach. (2011) J Thorac Oncol 6(11): 1784-1792.

105. Chua, K.N., Sim, W.J., Racine, V., et al. A cell-based small molecule screening method for identifying inhibitors of epithelial-mesenchymal transition in carcinoma. (2012) PloS One 7(3): e33183.

106. Fuchs, B.C., Fujii, T., Dorfman, J.D., et al. Epithelial-to-mesenchymal transition and integrin-linked kinase mediate sensitivity to epidermal growth factor receptor inhibition in human hepatoma cells. (2008) Cancer Res 68(7): 2391-2399.

107. Buck, E., Eyzaguirre, A., Rosenfeld-Franklin, M., et al. Feedback mechanisms promote cooperativity for small molecule inhibitors of epidermal and insulin-like growth factor receptors. (2008) Cancer Res 68(20): 8322-8332.

108. Shimono, Y., Mukohyama, J., Nakamura, S., et al. MicroRNA Regulation of Human Breast Cancer Stem Cells. (2015) J Clin Med $5(1)$.

109. Schwarzenbacher, D., Balic, M., Pichler, M. The role of microRNAs in breast cancer stem cells. (2013) Int J Mol Sci 14(7): 1471214723.

110. Ruan, K., Fang, X., Ouyang, G. MicroRNAs: novel regulators in the hallmarks of human cancer. (2009) Cancer Lett 285(2): 116-126.

111. Li, Q., Yao, Y., Eades, G., et al. Downregulation of miR-140 promotes cancer stem cell formation in basal-like early stage breast cancer. (2014) Oncogene 33(20): 2589-2600.

112. Gregory, P.A., Bert, A.G., Paterson, E.L., et al. The miR-200 family and miR-205 regulate epithelial to mesenchymal transition by targeting ZEB1 and SIP1. (2008) Nat Cell Biol 10(5): 593-601.

113. Eades, G., Yao, Y., Yang, M., et al. miR-200a regulates SIRT1 expression and epithelial to mesenchymaltransition (EMT)-like transformation in mammary epithelial cells. (2011) J Biol Chem 286(29): 25992-26002.

114. Rokavec, M., Wu, W., Luo, J.L. IL6-mediated suppression of miR200 c directs constitutive activation of inflammatory signaling circuit driving transformation and tumorigenesis. (2012) Mol Cell 45(6): 777789.
Ommega Online Publishers

Journal Title: International Journal of Cancer and Oncology (IJCO)

Journal Short Name: Int J Cancer Oncol
Journal ISSN: 2377-0902

E-mail: cancerandoncology@ommegaonline.com

Website: www.ommegaonline.org 